\title{
The effects of $n-3$ long-chain polyunsaturated fatty acids on bone formation and growth factors in adolescent boys
}

\author{
Camilla T. Damsgaard' ', Christian Mølgaard', Jeppe Matthiessen², Sedsel N. Gyldenløve' and Lotte Lauritzen'
}

INTRODUCTION: Animal studies indicate that n-3 long-chain polyunsaturated fatty acids (LCPUFAs) increase bone formation. To our knowledge, no studies have examined this in growing humans. This study investigated whether bone mass and markers of bone formation and growth were (i) associated with docosahexaenoic acid (DHA) status and (ii) affected by fish oil supplementation, in adolescent boys.

METHODS: Seventy-eight healthy, slightly overweight 13- to 15-y-old boys were randomly assigned to breads with DHA-rich fish oil (1.1 g/d n-3 LCPUFA) or control for $16 w k$. Whole-body bone mineral content $(B M C)$, bone area $(B A)$, bone mineral density (BMD), plasma osteocalcin, and growth factors were measured at wk 0 and wk 16, as well as diet, physical activity, and n-3 LCPUFA status in erythrocytes.

RESULTS: Fish oil strongly increased DHA status ( $P=0.0001)$. No associations were found between DHA status and BMC, BA, $\mathrm{BMD}$, or the markers of bone formation and growth at baseline. Furthermore, the fish oil intervention did not affect any of the outcomes as compared with control. However, dose-response analyses revealed a positive association between changes in DHA status and plasma insulin-like growth factor-1 (IGF-1) during intervention ( $\beta=0.24, P=0.03, n=78)$.

DISCUSSION: DHA status and fish oil supplementation were not associated with bone mass or markers of bone formation in adolescent boys, but IGF-1 increased with increasing DHA status.

B one mineral accumulation during childhood and adolescence plays an essential role in the prevention of osteoporosis (1). Bone growth peaks around 13-15 y of age in boys (2). During these $2 \mathrm{y}$, as much bone mineral is gained as will be lost during the bone mass decline in later adulthood (2). Although genetics explain about $70 \%$ of the variation in bone mass (3), modifiable factors such as diet and exercise are important targets in the prevention of osteoporosis and fractures (4).

Animal studies indicate that n-3 long-chain polyunsaturated fatty acids (LCPUFAs) from fatty fish increase markers of bone formation and reduce bone loss (5-9). A potential way in which n-3 LCPUFA may affect bone mineralization is via modulation of prostaglandin (PG) synthesis or regulation of expression of genes involved in cytokine production and osteoclast differentiation $(4,6)$. These experiments have mainly been performed in animal models for postmenopausal bone resorption (5-8). The majority of studies in young growing animals have supplemented with $\alpha$-linolenic acid, a plant-based n-3 PUFA (10), or n-3 LCPUFA in combination with n-6 PUFA (9,11-13), with limited effects on bone accumulation. A few randomized human trials, mainly in postmenopausal women, have investigated the impact of n-3 PUFA on bone mineral density (BMD) or biochemical markers of bone metabolism, with mixed results (14-16). A recent observational study in young Swedish men showed a positive association between docosahexaenoic acid (DHA) status, measured as percentage of total fatty acids in plasma, and bone mineral content (BMC) and BMD (17). This indicated a potential protective role of n-3 LCPUFA during growth. Fish intake is far below the national recommendations in Danish adolescents. Only about $10 \%$ of the boys reach the advised $200-300 \mathrm{~g}$ fish/wk (18), and therefore their n-3 LCPUFA intake and tissue status may be suboptimal.

Plasma osteocalcin, a protein secreted from bone osteoblasts, is used as a sensitive and specific biochemical marker of bone turnover and formation (19). In pubertal children, blood osteocalcin also correlates with linear growth velocity (19). Insulin-like growth factor-1 (IGF-1) is the main predictor of height velocity in childhood but has also been shown to be important for bone mineralization and bone strength $(20,21)$. More than $90 \%$ of the plasma IGF-1 is expected to be bound to IGF-binding protein-3 (IGF-BP3; ref. 22) and the IGF$1:$ IGF-BP3 ratio is thought to reflect the concentration of free, biologically active IGF-1 (ref. 20).

The aim of this study was to investigate whether BMC, bone area (BA), BMD, plasma osteocalcin, IGF-1, and IGF-BP3 were (i) associated with erythrocyte DHA status and (ii) affected by DHA-rich fish oil supplementation in healthy, slightly overweight adolescent boys. The article is based on the secondary outcomes of a trial investigating the effect of fish oil on cardiovascular risk markers in adolescent boys (23). 


\section{RESULTS}

\section{Subject Characteristics, Vitamin D Status, and Diet}

Subjects were successfully randomized with respect to anthropometry, habitual diet, pedometer-determined physical activity level, serum 25(OH)-vitamin D, and plasma testosterone (Table 1). The mean dietary intake of n-3 PUFA at baseline was about one-third of the recommended $1 \%$ of the energy intake $(\mathrm{E} \%)$ in the Nordic countries. The vitamin $D$ intake was about half of the recommended $7.5 \mu \mathrm{g} / \mathrm{d}$, whereas the mean calcium intake was just above the recommended $900 \mathrm{mg} / \mathrm{d}$ (ref. 24; Table 1). No changes were seen in these nutrients in the background diet in any of the groups during the intervention (data not shown). About one-third of the subjects had a serum $25(\mathrm{OH})$-vitamin $\mathrm{D}<25 \mathrm{nmol} / \mathrm{l}$ at the beginning of the study (November 2007-February $2008)$, indicating vitamin D deficiency $(25,26)$ and $6 \%$ had concentrations $<12.5 \mathrm{nmol} / 1$, indicating severe deficiency $(25,26)$. Serum 25(OH)-vitamin D did not change in either of the groups during the intervention $(P>0.60)$. As expected with puberty, plasma testosterone increased during the intervention from $30 \pm 3$ to $36 \pm 3 \mathrm{ng} / \mathrm{l}$ and from $35 \pm 3$ to $40 \pm$ $3 \mathrm{ng} / \mathrm{l}$ in the control and fish oil groups, respectively $(P<0.01$ in each of the groups), whereas no differences were seen between the groups at $16 \mathrm{wk}(P=0.56$ by analysis of covariance). No significant changes were observed in the intake of energy, macronutrients, saturated fatty acids, monounsaturated fatty acids, or PUFA in either of the groups during the

Table 1. Age, anthropometry, activity level, and dietary intake in the healthy boys at wk 0

\begin{tabular}{|c|c|c|}
\hline Characteristics & Control $(n=40)$ & Fish oil $(n=38)$ \\
\hline Age, y & $14.3 \pm 0.6^{\mathrm{a}}$ & $14.3 \pm 0.7$ \\
\hline Weight, kg & $66.6 \pm 9.9$ & $69.8 \pm 14.3$ \\
\hline Height, cm & $169 \pm 9$ & $169 \pm 11$ \\
\hline $\mathrm{BMI}, \mathrm{kg} / \mathrm{m}^{2}$ & $23.1(22.5-23.7)^{\mathrm{b}}$ & $24.1(23.1-25.2)$ \\
\hline $\begin{array}{l}\text { Percentage overweight/ } \\
\text { obese subjects }{ }^{c}\end{array}$ & $55 / 3$ & $50 / 16$ \\
\hline Activity level, steps/d & $10,950 \pm 3,842$ & $10,711 \pm 4253$ \\
\hline Plasma testosterone, ng/l & $30 \pm 17$ & $35 \pm 17$ \\
\hline $\begin{array}{l}\text { Serum } 25(\mathrm{OH}) \text {-vitamin D } \\
(\mathrm{nmol} / \mathrm{l})\end{array}$ & $30 \pm 16$ & $34 \pm 13$ \\
\hline \multicolumn{3}{|l|}{ Dietary intake } \\
\hline Energy, MJ/d & $9.3 \pm 2.5$ & $8.5 \pm 2.8$ \\
\hline Protein, E\% & $15 \pm 3$ & $16 \pm 3$ \\
\hline Carbohydrate, E\% & $51 \pm 5$ & $51 \pm 5$ \\
\hline Fat, E\% & $34 \pm 5$ & $33 \pm 5$ \\
\hline n-3 PUFA, E\% & $0.3 \pm 0.2$ & $0.3 \pm 0.2$ \\
\hline Vitamin $D, \mu \mathrm{g} / \mathrm{d}$ & $2.7 \pm 2.7$ & $3.1 \pm 3.1$ \\
\hline Calcium, mg/d & $1,049 \pm 479$ & $1,093 \pm 400$ \\
\hline \multicolumn{3}{|c|}{$\begin{array}{l}\mathrm{Cl} \text {, confidence interval; E\%, percentage of the energy intake; PUFA, polyunsaturated } \\
\text { fatty acid. }\end{array}$} \\
\hline \multicolumn{3}{|c|}{$\begin{array}{l}\text { aMean } \pm \text { SD (all such values). }{ }^{b} \text { Geometric mean }(95 \% \mathrm{Cl}) \text {. cBased on age- and sex-specific } \\
\text { cutoffs defined to pass through BMl of } 25 \text { and } 30 \mathrm{~kg} / \mathrm{m}^{2} \text { at age } 18 \text {, as according to } \\
\text { Cole et al. (40). }\end{array}$} \\
\hline
\end{tabular}

intervention (data not shown). However, sugar intake was reduced from $11 \pm 6$ to $9 \pm 5 \mathrm{E} \%$ and from $9 \pm 5$ to $7 \pm 5 \mathrm{E} \%$ in the control and fish oil groups, respectively $(P<0.05)$. This was probably a result of the dietary counseling in both groups and did not result in differences between the groups at wk 16 ( $P=0.18$ by analysis of covariance). During the intervention, the subjects grew $\sim 1.8 \mathrm{~cm}$ in height and gained $\sim 2.0 \mathrm{~kg}$ in both groups (23).

Cross-Sectional Associations of DHA-Status With BMC, BA, BMD, Size-Corrected BMC, and Markers of Growth and Bone Formation (wk 0)

The cross-sectional analysis at wk 0 did not show any associations between erythrocyte DHA and BMC, BA or BMD, IGF-1, IGF-BP3, or IGF-1:IGF-BP3 (Table 2). Size-corrected BMC was not associated with DHA status either $(B=0.01 \pm 0.01$, $P=0.49$ ). Inclusion of potential confounders and mediators as covariates in the multiple-regression models did not change the results (Table 2). Eicosapentaenoic acid (EPA) or total n-3 PUFA in erythrocytes was also not associated with any of the bone measurements or plasma markers (data not shown).

The adjusted models showed that plasma testosterone was a significant predictor of BMC, BA, BMD, IGF-1, and IGF1:IGF-BP3; plasma IGF-1 was a significant predictor of BMC, $\mathrm{BA}$, and osteocalcin; and plasma osteocalcin was a significant predictor of BMD, IGF-BP3, and IGF-1:IGF-BP3 (Table 2). Both plasma osteocalcin and testosterone were significant predictors of size-corrected BMC $(P<0.01)$. None of the other covariates were associated with any of the outcome variables in the adjusted models.

\section{Effects of 16-wk Fish Oil Supplementation on n-3 LCPUFA Status, BMC, BA, BMD, Size-Corrected BMC, and Markers of Growth and Bone Formation \\ Erythrocyte fatty acid composition did not differ between the fish oil and control groups at wk 0 (Table 3). As expected, the fish oil intervention strongly increased erythrocyte EPA and DHA levels and reduced erythrocyte n-6 PUFAs 18:2n-6 and 20:4n-6 as compared with control (Table 3). \\ Supplementation with fish oil for $16 \mathrm{wk}$ did not affect BMC, BA, BMD, osteocalcin, IGF-1, IGF-BP3, or IGF-1:IGF-BP3 levels as compared with control (Table 4), and size-corrected BMC level was not affected either $(P=0.17)$. Adjustment for changes in potential confounders and mediators did not change these results.}

\section{Dose-Response Associations Between Changes in n-3 LCPUFA Status With BMC, BA, BMD, size-corrected BMC, and Markers of Growth and Bone Formation \\ As the daily dose of fish oil varied between the subjects in the fish oil group, dose-response analyses were performed to investigate whether changes in DHA status during the inter- vention were associated with changes in the bone markers. In the total sample of subjects, erythrocyte DHA changes were positively associated with plasma IGF-1 (Figure 1). This asso- ciation was only slightly reduced after adjustment $(\beta=0.21$,}


$P=0.06, n=76)$. The regression coefficient was essentially unchanged when performed in only the fish oil group, but the $P$ value was reduced due to the reduced number of subjects $(\beta=0.26, P=0.12, n=38)$. No associations were seen between changes in erythrocyte DHA levels and changes in IGF-1 levels in the control group $(\beta=0.01, P=0.94, n=40)$. In the

Table 2. Associations of erythrocyte DHA status with bone mass and markers of growth and bone formation at wk 0 in the healthy boys

\begin{tabular}{lccl}
\hline Dependent variable & $B$ & SE & \multicolumn{1}{c}{$P$} \\
\hline BMC, g & 92.8 & 65.3 & 0.16 \\
Adjusted & 27.1 & 53.7 & $0.62^{\mathrm{a}, \mathrm{b}}$ \\
BA, cm ${ }^{2}$ & 45.5 & 39.3 & 0.25 \\
$\quad$ Adjusted & 11.0 & 32.4 & $0.74^{\mathrm{a}, \mathrm{b}}$ \\
BMD, g/cm ${ }^{2}$ & 0.02 & 0.01 & 0.15 \\
$\quad$ Adjusted & 0.01 & 0.01 & $0.63^{\mathrm{b}, \mathrm{c}}$ \\
Plasma IGF-1, $\mu \mathrm{g} / \mathrm{I}$ & 7.9 & 12.5 & 0.53 \\
Adjusted & -0.8 & 10.1 & $0.93^{\mathrm{b}, \mathrm{c}}$ \\
Plasma IGF-BP3, mg/l & -0.01 & 0.10 & 0.96 \\
$\quad$ Adjusted & -0.01 & 0.10 & $0.93^{\mathrm{c}}$ \\
IGF-1:IGF-BP3 & 1.4 & 1.7 & 0.40 \\
$\quad$ Adjusted & -0.1 & 1.3 & $0.93^{\mathrm{b}, \mathrm{c}}$ \\
Plasma osteocalcin, $\mu \mathrm{gg} / \mathrm{l}$ & -3.4 & 5.1 & 0.51 \\
$\quad$ Adjusted & -4.5 & 4.8 & $0.34^{\mathrm{a}}$ \\
\hline
\end{tabular}

$P$ value is shown for the partial regression coefficients (B) between erythrocyte DHA status (as independent variable) and bone markers (as dependent variables) obtained by multiple linear regression analysis, with or without adjustment for age, calcium intake, steps walked/d, serum testosterone, $25(\mathrm{OH})$-vitamin $\mathrm{D}$, and, if relevant, plasma osteocalcin and IGF-1; $n=77$ for IGF-1 and all adjusted models, $n=78$ in the remaining analyses.

$B A$, bone area: $B M C$, bone mineral content: $B M D$, bone mineral density: $D H A$, docosahexaenoic acid; IGF, insulin-like growth factor; IGF-BP3, IGF-binding protein-3. ${ }^{a}$ IGF- 1 was positively associated with the outcome variable, $P<0.02 .{ }^{\circ}$ Testosterone was positively associated with the outcome variable, $P<0.01$. 'Osteocalcin was positively associated with the outcome variable, $P<0.02$. total sample of boys $(n=78)$, changes in EPA levels were also positively associated with changes in IGF- 1 levels $(\beta=0.23$, $P=0.045, n=78)$ although not after adjustment $(\beta=0.15$, $P=0.18, n=78$ ). Comparable but nonsignificant associations were seen for total n-3 PUFA (data not shown). Also, changes in EPA and DHA levels tended to be positively associated with changes in IGF-BP3 levels $(\beta=0.24, P=0.03$ and $\beta=0.22$, $P=0.06$ for EPA levels before and after adjustment, respectively, and $\beta=0.20, P=0.08$ and $\beta=0.19, P=0.11$ for DHA levels before and after adjustment, respectively). No significant associations were seen between changes in IGF-1:IGFBP3 levels and changes in the n-3 PUFA status measures (data not shown).

\section{DISCUSSION}

This study showed no cross-sectional association between DHA status and bone mass evaluated by dual-energy X-ray absorptiometry (DXA) or biochemical markers of growth and bone formation in adolescent boys. This is in contrast with the findings of a recent article reporting positive correlations of plasma DHA and total n-3 PUFA with BMD in Swedish men at $22 \mathrm{y}$ of age (17). In that study, DHA and n-3 PUFA at $22 \mathrm{y}$ were also positively associated with changes in BMD at the spine from $16-22$ y of age. The sample size in that study $(n=78)$ was comparable to ours, but the participants in our trial were younger and slightly overweight. In contrast with the Swedish investigators, who measured n-3 PUFA status in plasma, we used erythrocytes. Erythrocytes reflect the n-3 PUFA intake during the past 3-5 mo and are therefore preferable when evaluating long-term status or habitual intake, whereas n-3 PUFA levels in plasma show large day-to-day variation and may thus reflect the last meals (27).

In the second part of our investigation, we found no effect of a 4-mo intervention with fish oil-enriched bread on biochemical markers of growth and bone formation or on bone mass evaluated by DXA. To our knowledge, this is the first randomized,

Table 3. Erythrocyte fatty acid composition in healthy boys receiving breads with fish oil or control at wk 0 , after 16 -wk intervention, and as changes during the intervention

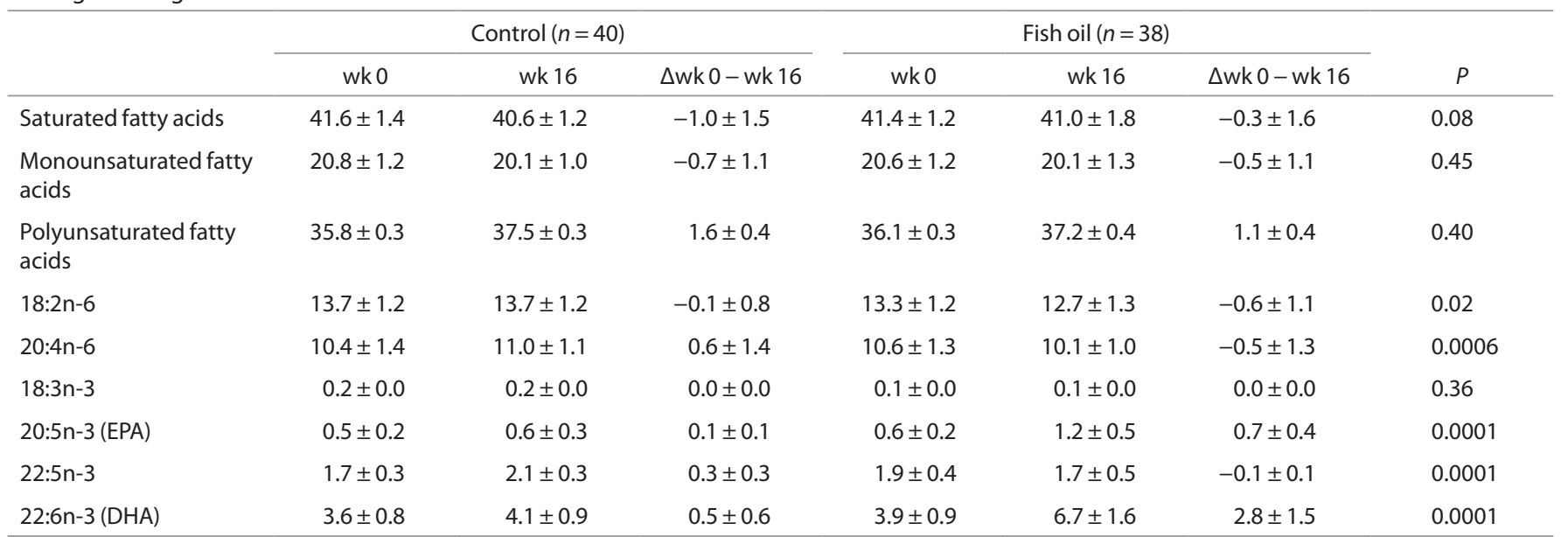

Data are presented in relative values (percentage of total fatty acids) as mean \pm SD. $P$ values indicate group differences at wk 16 , adjusted for wk-0 values in analysis of covariance. DHA, docosahexaenoic acid; EPA, eicosapentaenoic acid. Reprinted from ref. 23 with permission from Elsevier 2011. 
Table 4. Bone mass and markers of growth and bone formation in healthy boys receiving breads with fish oil or control at wk 0 , after 16 -wk intervention, and as changes during the intervention

\begin{tabular}{|c|c|c|c|c|c|c|c|c|}
\hline & \multicolumn{3}{|c|}{ Control $(n=40)$} & \multicolumn{3}{|c|}{ Fish oil $(n=38)$} & \multirow[b]{2}{*}{$P^{a}$} & \multirow[b]{2}{*}{$P_{\mathrm{adj}}^{\mathrm{b}}$} \\
\hline & wk 0 & wk 16 & $\Delta w k 0-w k 16$ & wk 0 & wk 16 & $\Delta \mathrm{wk} 0-\mathrm{wk} 16$ & & \\
\hline $\mathrm{BMC}(\mathrm{g})$ & $2,400 \pm 72$ & $2,515 \pm 75$ & $115 \pm 17$ & $2,482 \pm 92$ & $2,597 \pm 96$ & $115 \pm 16$ & 0.96 & 0.86 \\
\hline $\mathrm{BMD}\left(\mathrm{g} / \mathrm{cm}^{2}\right)$ & $1.07 \pm 0.01$ & $1.09 \pm 0.02$ & $0.01 \pm 0.00$ & $1.10 \pm 0.02$ & $1.12 \pm 0.02$ & $0.01 \pm 0.00$ & 0.96 & 0.94 \\
\hline Plasma osteocalcin ( $\mu \mathrm{g} / \mathrm{l})$ & $82 \pm 6$ & $88 \pm 6$ & $6 \pm 3$ & $82 \pm 7$ & $85 \pm 7$ & $4 \pm 4$ & 0.62 & 0.99 \\
\hline Plasma IGF-BP3 (mg/l)c & $5.5 \pm 0.2$ & $5.4 \pm 0.1$ & $-0.1 \pm 0.1$ & $5.4 \pm 0.1$ & $5.4 \pm 0.1$ & $0.1 \pm 0.1$ & 0.12 & 0.15 \\
\hline IGF-1:IGF-BP3 & $66 \pm 2$ & $66 \pm 2$ & $-0 \pm 1$ & $66 \pm 2$ & $67 \pm 2$ & $1 \pm 2$ & 0.56 & 0.59 \\
\hline
\end{tabular}

Data are presented as mean \pm SEM. wk 0 values were significant predictors of wk-16 values in all models $(P<0.001)$.

BA, bone area; BMC, bone mineral content; BMD, bone mineral density; IGF, insulin-like growth factor; IGF-BP3, IGF-binding protein-3.

a $P$ values indicate difference at wk 16 between control and fish oil group in analysis of covariance (ANCOVA) including wk-0 values as covariate. ${ }^{b} P_{\text {adj }}$ values indicate difference at wk 16 between control and fish oil group in ANCOVA including wk-0 values as covariate and with adjustment for age and changes in number of steps/d, calcium intake, serum testosterone, 25(OH)-vitamin D, plasma IGF-1, and osteocalcin. In these adjusted models, $n=39$ and $n=37$ in the control and fish oil groups, respectively, due to missing step reports in two participants. ${ }^{c} n=39$ for IGF-BP3 in the control group.

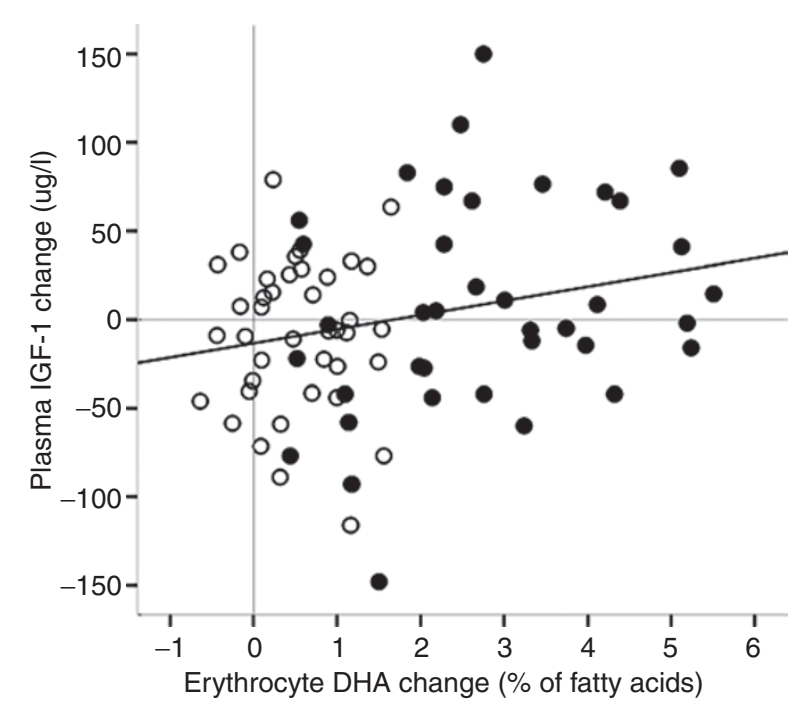

Figure 1. Dose-response association between changes in erythrocyte DHA-status and changes in plasma IGF-1 during the intervention in the total sample of 78 boys. Assignment to either the control group (open circles) or the fish oil group (filled circles) is indicated. The solid line represents the linear regression line $(\beta=0.24, P=0.03, n=78)$. DHA, docosahexaenoic acid; IGF-1, insulin-like growth factor-1.

controlled trial to investigate the effects of fish oil on bone mass in young growing individuals. Our findings agree with those of Bassey et al. (15), who supplemented pre- and postmenopausal women for $1 \mathrm{y}$ with fish oil + evening primrose oil (rich in the n-6 PUFA $\gamma$-linolenic acid) or control, and found no effect on $\mathrm{BMD}$ in either age group. Kruger et al. (14) gave calcium and either fish oil + evening primrose oil or coconut oil (control) to osteoporotic women for $18 \mathrm{mo}$ and observed a larger decline in lumbar and femoral BMD in the control group as compared with the PUFA group. However, in these two trials the effect of n-3 PUFA could not be separated from that of n-6 PUFA. Appleton et al. (28) supplemented mild-moderately depressed adults (18-67 y) with n-3 LCPUFA or olive oil (control) for $12 \mathrm{wk}$, and found no effect on serum $\beta$-C-terminal telopeptide, a marker of bone resorption. Reductions in another bone resorption marker, $\mathrm{N}$-telopeptides, were seen in a three-period 6-wk crossover study, after consumption of a diet rich in $\alpha$-linolenic acid, but not linoleic acid, as compared with control (average American diet; ref. 16). None of these trials measured bone mass, e.g., by DXA scan. Moreover, the interaction of n-3 LCPUFA with bone turnover may be different in osteoporotic as compared with healthy subjects. Altogether, these trials aimed at reducing the age-associated bone mineral loss, whereas we investigated the potential of fish oil to increase growth-associated markers of bone accumulation during a period of high growth.

IGF-1 is known to be an important mediator of growth hormone in children and adolescents, affecting both linear growth and bone mass acquisition $(21,29)$. Our dose-response analyses showed positive associations between changes in DHA status and changes in IGF-1 and positive associations between IGF-1 and size-corrected BMC. This may indicate that fish oil dose-dependently modulates bone growth signaling but that the effects on actual bone mass were not yet manifested after just $4 \mathrm{mo}$, despite the very rapid growth of our subjects. To our knowledge, the effects of n-3 LCPUFA on IGF-1 have not been investigated earlier in humans. In growing rats, Green et al. (30) found no effect of fish oil vs. corn oil on IGF-1, whereas femur BMD was increased with fish oil. As mentioned earlier, the IGF1:IGF-BP3 ratio has been proposed as an index of bioactive IGF-1 (22), but this was not affected by fish oil or DHA status in our study. The implications of this remain to be elucidated.

Different mechanisms for the effect of n-3 LCPUFA have been proposed. When n-6 PUFAs are substituted for n-3 LCPUFAs in the diet, a shift in PG production from $\mathrm{PGE}_{2}$ to $\mathrm{PGE}_{3}$ is thought to occur (12). $\mathrm{PGE}_{2}$ has been shown to stimulate bone resorption, and fish oil consumption would thus be speculated to reduce bone breakdown, through a reduction in $\mathrm{PGE}_{2}$ concentrations, as shown in rats (12). However, depending on its concentration, $\mathrm{PGE}_{2}$ has also been shown to induce IGF-1 and thereby evoke positive effects on bone formation (31). n-3 LCPUFAs have also been demonstrated to reduce the 
production of inflammatory cytokines that stimulate osteoclast differentiation and bone resorption (4). However, a reduction in cytokine production with fish oil consumption has been difficult to demonstrate consistently in healthy humans (32).

A high n-3 LCPUFA status at baseline could potentially explain the lack of effect of fish oil on bone mineralization in this study. However, in our study population, the total n-3 PUFA intake was one-third of the recommended intake, and the erythrocyte content of EPA and DHA before intervention was low as compared to that of young Finnish men (33), although slightly higher than that of American 6- to 13-y-old children (34). Also, in the Swedish study mentioned earlier (17), associations between n-3 LCPUFA status and BMD were seen despite a fairly high plasma n-3 LCPUFA status. Another important explanatory variable could be the n-3 LCPUFA dose. The median EPA + DHA dose in this study was $1.1 \mathrm{~g} / \mathrm{d}$, which was sufficient to reduce blood pressure in our subjects, as earlier reported (23). Previous trials of n-3 LCPUFA on bone in adults have used both lower and higher doses $(14,15,28)$. The dose differences do not correlate with differences in the outcomes of the trial, as the only one that showed an effect used a much lower dose than ours (14). Moreover, as compared with the other studies, our fish oil had a very high content of DHA, which is thought to be the most important bioactive n-3 LCPUFA in bone modeling, whereas the other studies used EPA-rich supplements.

We performed all our statistical analyses with and without adjustment for a number of potential confounders and modifying factors, and our results proved to be highly consistent among the different models. Adjustment for physiological maturity status is important in adolescents, as the maturityrelated physiological changes occurring during growth can vary considerably at the same chronological age (1). Moreover, sex hormones are known to directly affect acquisition of bone strength (29). We used plasma testosterone concentrations as a measure of maturity status. Self-evaluated puberty status, e.g., by Tanner stages, may have been preferred, but during the planning of the study this method was judged to be too intimidating in this particular population.

In conclusion, this randomized, controlled trial is the first to investigate the effects of fish oil on bone formation and growth factors in adolescent boys. First, our cross-sectional analysis at baseline showed no associations between erythrocyte DHA or n-3 PUFA status with bone mass measured by DXA or markers of growth and bone formation in healthy, slightly overweight adolescent boys. Second, 16-wk intervention with DHA-rich fish oil did not affect any of these measures in this population. However, dose-response analyses indicated that plasma IGF-1 increased with DHA status during the intervention. This could potentially have bone-accumulating consequences, which should be investigated further in randomized trials over longer time spans.

\section{METHODS}

\section{Study Design and Subjects}

The study protocol was approved by the Committee of Biomedical Research Ethics in the Capital Region of Denmark (H-A-2007-0055) and registered in the database clinicaltrials.gov (no. NCT00929552).
The study had a randomized, double-blind, parallel design and was performed from November 2007 through June 2008. The details of the study procedures were published elsewhere (23). In brief, subjects were recruited through the Danish Civilian Person Register and a total of 3,652 boys aged 13-15 y were invited by letter. Inclusion criterion was self-reported BMI above the 90th percentile based on a Danish reference population (35). Exclusion criteria were regular smoking or chronic diseases except mild allergies. Informed written consent was obtained from the boys and their parents, and 78 of the 87 subjects who entered the trial completed the study. Reasons for withdrawal included dislike of the intervention breads and inability to eat the instructed daily amount of bread. Apart from the breads, brief individual lifestyle counseling toward healthier diets and increased physical activity were provided to all subjects every $2 \mathrm{wk}$ during the intervention. The counseling was based on the National Danish Dietary Guidelines (36) and the results of each subject's 4-d precoded dietary records and 7-d physical activity recording with a pedometer (SW-200; Yamax, Tokyo, Japan) before the start of the intervention (23). These recordings of diet and physical activity were repeated at the end of the 16-wk intervention period.

\section{Dietary Intervention}

Subjects were randomly assigned to intervention breads with either fish oil or control oil for $16 \mathrm{wk}$. Oils were baked into breads by Kohberg $\mathrm{A} / \mathrm{S}$ (Bolderslev, Denmark), and subjects were asked to consume two pieces of rye bread and one wheat roll per day. A DHA-rich granulate of tuna oil (NuMega; Clover, Sydney, Australia) was added to the bread and the control oil was a 6:1:1 mix of palm shortening, soy oil, and rapeseed oil, designed to reflect the fatty acid composition in a typical Danish diet. Expressed as a percentage of total fatty acids, the fish oil group received bread with $24.9 \% \mathrm{n}-3$ PUFA (mainly as DHA) and $13.5 \% \mathrm{n}-6$ PUFA (mainly as linoleic acid), whereas the control group received bread with $1.9 \% \mathrm{n}$-3 PUFA (mainly as $\alpha$-linolenic acid) and $28.0 \%$ n-6 PUFA (mainly as linoleic acid). Overall the fish oil breads had a higher PUFA content and a lower content of monounsaturated fatty acids than the control breads (23). The daily ration of bread supplied a median of $4.9 \mathrm{~g}$ (range, $4.1-5.4 \mathrm{~g}$ ) fat in the fish oil group and $4.2 \mathrm{~g}$ (range, $3.0-5.0 \mathrm{~g}$ ) fat in the control group, with $\mathrm{n}-6 / \mathrm{n}-3$ PUFA ratios of 0.5 and 14.5 , respectively (23). Overall, in the fish oil group the breads supplied $191 \mathrm{mg} / \mathrm{d} \mathrm{EPA}+892 \mathrm{mg} / \mathrm{d}$ DHA. Self-reported compliance with the breads was 90\% (range, 70-100\%) in both groups. The n-3 LCPUFA content of erythrocytes was used as another measure of compliance.

\section{Anthropometry and DXA}

The subjects were examined at wk 0 (baseline) and after the 16-wk intervention. Height was measured to the nearest $\mathrm{cm}$, and weight to the nearest $100 \mathrm{~g}$ on an electronic scale (Frederiksberg Vægtfabrik, Frederiksberg, Denmark). The subjects underwent a whole-body DXA scan in a Lunar Prodigy GE scanner (Madison, WI). Subjects were scanned in light clothes and were told to remove all pieces of metal from their bodies. The scanner had a reported precision of $0.9 \%$ (37) and a radiation dose of $1.2 \mu \mathrm{Sv}$, corresponding to about $4 \mathrm{~h}$ background radiation in Denmark. Based on double scans performed in adults during $5 \mathrm{mo}$, the coefficients of variation were $1.4 \%$ and $0.5 \%(n=18)$ for whole-body BMC and BMD, respectively. Body 
size-corrected BMC was calculated as BMC adjusted for BA, weight, and height, after natural logarithm transformation of the variables, as described by Prentice et al. (38).

\section{Blood Samples}

At both visits, blood was drawn in the morning after an overnight fast from the antecubital vein in lithium-heparin coated tubes for analysis of erythrocyte fatty acid composition, IGF-1 and IGF-BP3 levels, and the bone formation marker osteocalcin. Uncoated tubes were used for analysis of serum $25(\mathrm{OH})$-vitamin $\mathrm{D}$ and testosterone. Blood was kept on ice until centrifugation at $2500 \mathrm{~g}, 4^{\circ} \mathrm{C}$ for $10 \mathrm{~min}$. Erythrocytes were washed three times in isotonic saline solution, reconstituted 1:1 in isotonic saline with $0.005 \%$ butylated hydroxytoluene, and blown with nitrogen. All samples were stored at $-80^{\circ} \mathrm{C}$ until analysis. The fatty acid composition of erythrocytes and intervention breads was determined by gas chromatography as described previously (23) and expressed as percentage of total fatty acids. Plasma osteocalcin, testosterone, IGF-1, and IGF-BP3 concentrations were measured on an Immulite 1000 (Siemens, Los Angeles, CA). Serum 25(OH)-vitamin D was analyzed with a Vitamin D External Quality Assessment Scheme-accredited fully automated direct competitive chemiluminescent immunoassay (Liaison 25-OH Vitamin D TOTAL; DiaSorin, Stillwater, MN). Within-run and total precision of the vitamin D analysis were $2.9-5.5 \%$ and $6.3-12.9 \%$, respectively (39).

\section{Statistical Analysis}

Normal distribution was checked with the Shapiro-Wilk's test and visual inspection of histograms. Changes in diet, serum 25(OH)-vitamin $\mathrm{D}$, and plasma testosterone in each group during the intervention were tested by paired $t$-test. Multiple linear regression analysis was performed on wk-0 measurements to assess associations of erythrocyte DHA status with the bone measurements (BMC, BA, BMD, and size-corrected BMC) and biochemical markers (osteocalcin, IGF-1, IGF-BP3, and IGF-1:IGF-BP3), and to evaluate the potential modifying effects of age, calcium intake, pedometer-determined physical activity level, serum testosterone, $25(\mathrm{OH})$-vitamin $\mathrm{D}$, plasma osteocalcin, and IGF-1 (where appropriate). The intervention effects of fish oil vs. control on the bone measurements and biochemical markers were analyzed by analysis of covariance with wk-16 measurements as outcomes and wk- 0 measurements as covariates. The analyses were performed with and without adjustment for age and for changes in number of steps/d, calcium intake, plasma osteocalcin, IGF-1, serum 25(OH)-vitamin D, and testosterone. Finally, to look for doseresponse effects of fish oil, changes in the bone measurements and biochemical markers were analyzed as a function of changes in erythrocyte DHA status in multiple linear regression analysis. These analyses were performed with and without adjustment for changes in the aforementioned covariates, except for age, which was entered in the model with its value at wk 0 . Data were analyzed with SPSS software (version 18; SPSS, Chicago, IL). Numbers in the text are presented as means $\pm \mathrm{SD}$, unless otherwise stated. A total sample size of $n=80$ was calculated to give sufficient power to detect differences between the control and fish oil groups of $0.6 \mathrm{SD}$ in the outcome variables. For our osteocalcin measurements, this means that we would be able to detect differences between the two groups in osteocalcin changes of $<15-23 \mu \mathrm{g} / \mathrm{l}$.

\section{ACKNOWLEDGMENTS}

We thank Maiken H. Pedersen, Julie Brønholm, Birgitte Hermansen, and Helle Frederiksen for collecting the data, Ümmühan Celik for sampling the blood, Pia Madsen and Trang Vu for performing the fatty acid analyses, and Henrik Krarup (Department of Clinical Biochemistry, Aalborg University Hospital, Denmark) for performing the vitamin D analyses.

\section{STATEMENT OF FINANCIAL SUPPORT}

This study was supported by the Danish Council for Strategic Research. Intervention breads were sponsored by Kohberg A/S, Bolderslev, Denmark.

\section{REFERENCES}

1. MacKelvie KJ, Khan KM, McKay HA. Is there a critical period for bone response to weight-bearing exercise in children and adolescents? a systematic review. Br J Sports Med 2002;36:250-7; discussion 257.

2. Bailey DA. The Saskatchewan Pediatric Bone Mineral Accrual Study: bone mineral acquisition during the growing years. Int J Sports Med 1997;18:Suppl 3:S191-4.

3. Eisman JA. Genetics of osteoporosis. Endocr Rev 1999;20:788-804.

4. Salari P, Rezaie A, Larijani B, Abdollahi M. A systematic review of the impact of $\mathrm{n}-3$ fatty acids in bone health and osteoporosis. Med Sci Monit 2008;14:RA37-44.

5. Watkins BA, Li Y, Seifert MF. Dietary ratio of n-6/n-3 PUFAs and docosahexaenoic acid: actions on bone mineral and serum biomarkers in ovariectomized rats. J Nutr Biochem 2006;17:282-9.

6. Sun D, Krishnan A, Zaman K, Lawrence R, Bhattacharya A, Fernandes G. Dietary n-3 fatty acids decrease osteoclastogenesis and loss of bone mass in ovariectomized mice. J Bone Miner Res 2003;18:1206-16.

7. Shen CL, Yeh JK, Rasty J, et al. Improvement of bone quality in gonadintact middle-aged male rats by long-chain n-3 polyunsaturated fatty acid. Calcif Tissue Int 2007;80:286-93.

8. Shen CL, Yeh JK, Rasty J, Li Y, Watkins BA. Protective effect of dietary long-chain n-3 polyunsaturated fatty acids on bone loss in gonad-intact middle-aged male rats. Br J Nutr 2006;95:462-8.

9. Watkins BA, Shen CL, McMurtry JP, et al. Dietary lipids modulate bone prostaglandin E2 production, insulin-like growth factor-I concentration and formation rate in chicks. J Nutr 1997;127:1084-91.

10. Cohen SL, Ward WE. Flaxseed oil and bone development in growing male and female mice. J Toxicol Environ Health Part A 2005;68:1861-70.

11. Weiler HA, Fitzpatrick-Wong S. Dietary long-chain polyunsaturated fatty acids minimize dexamethasone-induced reductions in arachidonic acid status but not bone mineral content in piglets. Pediatr Res 2002;51:282-9.

12. Kelly O, Cusack S, Jewell C, Cashman $\mathrm{KD}$. The effect of polyunsaturated fatty acids, including conjugated linoleic acid, on calcium absorption and bone metabolism and composition in young growing rats. Br J Nutr 2003;90:743-50.

13. Mollard RC, Kovacs HR, Fitzpatrick-Wong SC, Weiler HA. Low levels of dietary arachidonic and docosahexaenoic acids improve bone mass in neonatal piglets, but higher levels provide no benefit. J Nutr 2005;135:505-12.

14. Kruger MC, Coetzer H, de Winter R, Gericke G, van Papendorp DH. Calcium, gamma-linolenic acid and eicosapentaenoic acid supplementation in senile osteoporosis. Aging (Milano) 1998;10:385-94.

15. Bassey EJ, Littlewood JJ, Rothwell MC, Pye DW. Lack of effect of supplementation with essential fatty acids on bone mineral density in healthy preand postmenopausal women: two randomized controlled trials of Efacal v. calcium alone. Br J Nutr 2000;83:629-35.

16. Griel AE, Kris-Etherton PM, Hilpert KF, Zhao G, West SG, Corwin RL. An increase in dietary n-3 fatty acids decreases a marker of bone resorption in humans. Nutr J 2007;6:2.

17. Högström M, Nordström P, Nordström A. n-3 Fatty acids are positively associated with peak bone mineral density and bone accrual in healthy men: the NO2 Study. Am J Clin Nutr 2007;85:803-7.

18. National Food Institute. Dietary habits in Denmark 2003-2008 - Main Results [Danskernes Kostvaner 2003-2008 - Hovedresultater]. Søborg, Denmark: Rosendahls - Schultz Grafisk, 2010:1-196.

19. Szulc P, Seeman E, Delmas PD. Biochemical measurements of bone turnover in children and adolescents. Osteoporos Int 2000;11:281-94.

20. Juul A. Serum levels of insulin-like growth factor I and its binding proteins in health and disease. Growth Horm IGF Res 2003;13:113-70. 
21. Yakar S, Canalis E, Sun H, et al. Serum IGF-1 determines skeletal strength by regulating subperiosteal expansion and trait interactions. J Bone Miner Res 2009;24:1481-92.

22. Juul A, Dalgaard P, Blum WF, et al. Serum levels of insulin-like growth factor (IGF)-binding protein-3 (IGFBP-3) in healthy infants, children, and adolescents: the relation to IGF-I, IGF-II, IGFBP-1, IGFBP-2, age, sex, body mass index, and pubertal maturation. J Clin Endocrinol Metab 1995;80:2534-42.

23. Pedersen MH, Mølgaard C, Hellgren LI, Lauritzen L. Effects of fish oil supplementation on markers of the metabolic syndrome. J Pediatr 2010;157:395-400, 400.e1.

24. Nordic Council of Ministers. Nordic Nutrition Recommendations 2004. Copenhagen, Denmark: Norden, 2004:1-436.

25. Misra M, Pacaud D, Petryk A, Collett-Solberg PF, Kappy M; Drug and Therapeutics Committee of the Lawson Wilkins Pediatric Endocrine Society. Vitamin D deficiency in children and its management: review of current knowledge and recommendations. Pediatrics 2008;122: 398-417.

26. Wharton B, Bishop N. Rickets. Lancet 2003;362:1389-400.

27. Katan MB, Deslypere JP, van Birgelen AP, Penders M, Zegwaard M. Kinetics of the incorporation of dietary fatty acids into serum cholesteryl esters, erythrocyte membranes, and adipose tissue: an 18-month controlled study. J Lipid Res 1997;38:2012-22.

28. Appleton KM, Fraser WD, Rogers PJ, Ness AR, Tobias JH. Supplementation with a low-moderate dose of $\mathrm{n}-3$ long-chain PUFA has no short-term effect on bone resorption in human adults. Br J Nutr 2011;105:1145-9.

29. Davies JH, Evans BA, Gregory JW. Bone mass acquisition in healthy children. Arch Dis Child 2005;90:373-8.

30. Green KH, Wong SC, Weiler HA. The effect of dietary n-3 long-chain polyunsaturated fatty acids on femur mineral density and biomarkers of bone metabolism in healthy, diabetic and dietary-restricted growing rats. Prostaglandins Leukot Essent Fatty Acids 2004;71:121-30.
31. McCarthy TL, Centrella M, Raisz LG, Canalis E. Prostaglandin E2 stimulates insulin-like growth factor I synthesis in osteoblast-enriched cultures from fetal rat bone. Endocrinology 1991;128:2895-900.

32. Sijben JW, Calder PC. Differential immunomodulation with long-chain n-3 PUFA in health and chronic disease. Proc Nutr Soc 2007;66:237-59.

33. Vidgren HM, Agren JJ, Schwab U, Rissanen T, Hänninen O, Uusitupa MI. Incorporation of n-3 fatty acids into plasma lipid fractions, and erythrocyte membranes and platelets during dietary supplementation with fish, fish oil, and docosahexaenoic acid-rich oil among healthy young men. Lipids 1997;32:697-705.

34. Stevens L, Zhang W, Peck L, et al. EFA supplementation in children with inattention, hyperactivity, and other disruptive behaviors. Lipids 2003;38:1007-21.

35. Andersen E, Hutchings B, Jansen J, Nyholm M. [Heights and weights of Danish children]. Ugeskr Laeg 1982;144:1760-5.

36. The Danish Nutrition Council and National Food Institute. National Danish Dietary Guidelines [Kostrådene 2005]. Report no. 36. Søborg, Denmark: Boje \& Mobeck, 2011:1-88.

37. Williams JE, Wells JC, Wilson CM, Haroun D, Lucas A, Fewtrell MS. Evaluation of Lunar Prodigy dual-energy X-ray absorptiometry for assessing body composition in healthy persons and patients by comparison with the criterion 4-component model. Am J Clin Nutr 2006;83:1047-54.

38. Prentice A, Parsons TJ, Cole TJ. Uncritical use of bone mineral density in absorptiometry may lead to size-related artifacts in the identification of bone mineral determinants. Am J Clin Nutr 1994;60:837-42.

39. Wagner D, Hanwell HE, Vieth R. An evaluation of automated methods for measurement of serum 25-hydroxyvitamin D. Clin Biochem 2009;42:1549-56.

40. Cole TJ, Bellizzi MC, Flegal KM, Dietz WH. Establishing a standard definition for child overweight and obesity worldwide: international survey. BMJ 2000;320:1240-3. 